\title{
Ionic liquid/ultrasound pretreatment and in situ enzymatic saccharification of bagasse using biocompatible cholinium ionic liquid
}

Kazuaki Ninomiya ${ }^{\mathrm{a}}$, Asami Kohori ${ }^{\mathrm{b}}$, Mai Tatsumi ${ }^{\mathrm{b}}$, Koji Osawa ${ }^{\mathrm{b}}$, Takatsugu Endo ${ }^{\mathrm{b}}$, Ryohei Kakuchi ${ }^{\mathrm{b}}$, Chiaki Ogino ${ }^{\mathrm{c}}$, Nobuaki Shimizu ${ }^{\mathrm{a}}$, and Kenji Takahashi ${ }^{\mathrm{b}}$ **

${ }^{a}$ Institute of Nature and Environmental Technology,

Kanazawa University, Kakuma-machi, Kanazawa 920-1192, Japan

${ }^{b}$ Faculty of Natural System, Institute of Science and Engineering, Kanazawa University, Kakuma-machi, Kanazawa 920-1192, Japan

${ }^{c}$ Department of Chemical Science and Engineering, Graduate School of Engineering, Kobe University, 1-1 Rokkodaicho, Nada-ku, Kobe 657-8501, Japan

* Corresponding author.

Tel.:+81 76234 4828; fax:+81 762344829

E-mail address: ktkenji@staff.kanazawa-u.ac.jp (K. Takahashi) 


\begin{abstract}
Choline acetate (ChOAc), a cholinium ionic liquid (IL), showed almost the same bagasse pretreatment capability as 1-ethyl-3-methylimidazolium acetate (EmimOAc), a conventional imidazolium IL used for biomass pretreatment. Moreover, ChOAc showed less of an inhibitory effect on cellulase than EmimOAc. Thus, ChOAc was used for IL/ultrasound-assisted pretreatment and in situ enzymatic saccharification, where IL was not washed out from the pretreated bagasse but diluted with the addition of a buffer solution. When in situ saccharification was performed for $48 \mathrm{~h}$ in the presence of $10 \%$ ChOAc, the cellulose and hemicellulose saccharification percentages were $80 \%$ and 72\%, respectively. When ChOAc was increased to 20\%, the saccharification percentages were $72 \%$ and $53 \%$, respectively. However, the values were just $28 \%$ and 2\%, respectively, in case of 20\% EmimOAc. A glucose/xylose solution free from IL and ChOAc aqueous solution without these sugars could be recovered separately by electrodialysis of the hydrolysate of in situ saccharification.
\end{abstract}

\title{
Keywords:
}

Cholinium, Ionic liquid, Pretreatment, in-situ saccharification, Lignocellulose. 


\section{Introduction}

Ionic liquids (ILs), generally defined as organic salts that melt below $100^{\circ} \mathrm{C}$, have received much attention of late, because they are thermally stable, nonvolatile, and capable of dissolving various polymeric compounds even under mild conditions. A previous study demonstrated that ILs can dissolve cellulose (Swatloski, et al., 2002), and that cellulose re-precipitated after being dissolved in ILs exhibits a much greater efficiency of enzymatic hydrolysis due to its decreased crystallinity (Dadi, et al., 2006). Moreover, several groups have extended this IL-assisted pretreatment method to various lignocellulosic biomasses (Brandt, et al., 2013). It has been demonstrated that the IL-assisted pretreatment methods are more effective than the conventional methods using diluted acid or ammonia (Li, et al., 2010; Li, et al., 2011).

In the IL-assisted pretreatment process, the pretreated biomass has to be washed extensively so as to remove the residual IL because the residual IL in pretreated biomass causes inhibition of cellulolytic enzymes and fermentative microorganisms during saccharification and fermentation (Datta, et al., 2010; Ouellet, et al., 2011). However, this extensive washing of pretreated biomass results in large amounts of diluted IL aqueous solution, which leads to a high cost for concentrating IL from its diluted aqueous solution by evaporation and for treating the resultant wastewater. The cost issue can be an obstacle for scale-up of IL-assisted pretreatment processes.

Recently, researchers have suggested a simple process comprising IL-assisted pretreatment and in situ enzymatic saccharification without washing out IL from the pretreated biomass and with the addition of a smaller amount of water for diluting IL in order to save costs derived from the extensive washing step (Kamiya, et al., 2008; Yang, et al., 2010; Li, et al., 2010; Feng, et al., 2011; Wang, et al., 2011; Li, et al, 2012; Abels, 
et al, 2013; Shi, et al, 2013; Xu, et al., 2014). Among these, some reports further demonstrated the separation of a monomeric sugar solution and an IL aqueous solution after in situ enzymatic saccharification (Li, et al., 2010; Feng, et al., 2011; Li, et al., 2012; Abels, et al., 2013; Shi, et al., 2013). To reduce the water use for diluting IL and to achieve the higher sugar yield/concentration in the in situ enzymatic saccharification, the following factors are important: 1) high loading of biomass to IL (less IL amount per unit biomass) during the IL pretreatment step, reducing water use per unit biomass; 2) use of biocompatible IL and/or IL-tolerant cellulase enzymes, which reduce water use for diluting IL before enzymatic saccharification because a higher IL concentration is possible. Some in situ enzymatic saccharification has even been performed by employing biocompatible IL such as 1-ethyl-3-methylimidazolium diethylphosphate (EmimDEP) (Kamiya, et al., 2008), 1,3-dimethylimidazolium dimethylphosphate (MmimDMP) (Yang, et al., 2010; Li, et al., 2010; Feng, et al., 2011; Abels, et al., 2013), as well as conventional IL such as 1-ethyl-3-methylimidazolium acetate (EmimOAc) (Wang, et al., 2011; Shi, et al., 2013). Others employed the IL tolerant cellulase (Shi, et al., 2013). Moreover, recently, both biocompatible IL [1-ethyl-3-methylimidazolium dimethylphosphate (EmimDMP)] and IL tolerant cellulase were used simultaneously (Xu, et al., 2014). However, to the best of our knowledge, all IL used for in situ saccharification was limited to the imidazolium IL.

Completely bio-derived cholinium ILs were reported in 2007 that contain cholinium cations combined with amino acid-based anions (Hu, et al., 2007) or carboxylic acid-based anions (Fukaya, et al., 2007). In previous studies, it was reported that these cholinium ILs could be used for pretreatment to enhance the enzymatic hydrolysis of lignocellulosic material (Liu, et al., 2012; Ninomiya, et al., 2013a,b,c). 
Moreover, our previous study demonstrated that choline acetate (ChOAc) was less toxic for fermentative microorganisms than EmimOAc (Ninomiya, et al., 2013b). However, to the best of our knowledge, there is no report on in situ enzymatic saccharification using cholinium IL as a candidate for biocompatible IL.

Therefore in the present study, we employ ChOAc as a biocompatible cholinium IL in order to demonstrate the IL pretreatment and in situ enzymatic saccharification of lignocellulosic biomass. The data for pretreatment capability, inhibition to commercial cellulase, and performance of the in situ saccharification process were then compared with data obtained using the standard imidazolium IL, EmimOAc. Moreover, we have demonstrated electrodialysis-assisted separation of IL and monomeric sugar obtained by in situ enzymatic saccharification.

\section{Materials and Methods}

\subsection{Biomass, IL, and cellulase}

Bagasse powder (approximately $<3 \mathrm{~mm}$ ) was purchased from Sanwa Cellulosin Co., Ltd. (Yokkaichi, Japan) as the lignocellulosic material. The bagasse powder was ground by a mill and then sieved to obtain a powder of 250-500 $\mu$ m. Microcrystalline

cellulose (Avicel ${ }^{\circledR} \mathrm{PH}-101$ ) was purchased from Sigma-Aldrich (St. Louis, MO, USA). Phosphoric acid swollen cellulose (PASC) was prepared from the microcrystalline cellulose according to the method described previously (Wood, 1988).

Choline acetate (ChOAc) was prepared by a one-pot neutralization method (Yu, et al., 2008) with minor modifications. An equimolar amount of acetic acid was added dropwise to a choline hydroxide solution (45 wt\%) in methanol (Sigma-Aldrich) with cooling. The mixture was stirred at room temperature (RT) for $6 \mathrm{~h}$. Water and methanol 
were removed in vacuo using a rotary evaporator at $40^{\circ} \mathrm{C}$ for $1 \mathrm{~h}$ and then $90^{\circ} \mathrm{C}$ for $2 \mathrm{~h}$. The resultant residue was dried under a vacuum at RT for $16 \mathrm{~h}$. The water content for ChOAc was measured below 0.5 wt\% by Karl-Fischer titration (AQ-2200, Hiranuma Sangyo Co., Ltd, Mito, Japan). 1-ethyl-3-methylimidazolium acetate (EmimOAc) was purchased from Kanto Chemical Co., Inc. (Tokyo, Japan).

A commercial cellulase Cellic ${ }^{\circledR}$ CTec2, a complex blend of cellulase, hemicellulose, and $\beta$-glucosidase, (batch number VCNI 0008, 106 filter paper units (FPU) per milliliter) was purchased from Novozymes (Franklinton, NC, USA). The FPU of the cellulase was determined by the method reported by NREL (Adney and Baker, 2008). All other chemicals were from commercial sources and of reagent grade.

\subsection{IL-assisted pretreatment and in situ enzymatic saccharification}

Bagasse was pretreated with IL and then hydrolyzed enzymatically without washing out IL from the treated bagasse. For the pretreatment step, $0.25 \mathrm{~g}$ of bagasse powder was added to a $15 \mathrm{~mL}$ Corning tube containing $5 \mathrm{~g}$ of IL (EmimOAc or ChOAc). The biomass/IL mixture in the tube was sonicated, since our previous study revealed that sonication was more effective than conventional heating (Ninomiya, et al., 2013c). The ultrasound was irradiated for $60 \mathrm{~min}$ at $24 \mathrm{kHz}$ and an emission power of $35 \mathrm{~W}$ using an ultrasonic processor (UP201S with sonotrode S3, Hielscher Ultrasonics GmbH, Teltow, Germany) in a water bath maintained at $25^{\circ} \mathrm{C}$. Bagasse powder without the IL pretreatment was used as a negative control sample, when necessary.

For the subsequent step of enzymatic hydrolysis, the biomass/IL mixture was diluted with $45,20,11.7$, and $7.5 \mathrm{~mL}$ of $50 \mathrm{mM}$ phosphate buffer $(\mathrm{pH}=5.0)$ to adjust the IL concentration to 10, 20, 30, and $40 \mathrm{wt} \%$. For the positive control, IL was washed 
out from the pretreated biomass. The biomass/IL mixture was diluted with $45 \mathrm{~mL}$ of deionized water in a 50-mL tube, which resulted in the precipitation of the biomass. After stirring, the $50-\mathrm{mL}$ tube was centrifuged $(8,000 \times \mathrm{g})$ for $10 \mathrm{~min}$ at $25^{\circ} \mathrm{C}$, and the supernatant was removed. The washing procedure was repeated five times to remove IL. Then, the biomass was re-suspended in $5 \mathrm{~mL}$ of the phosphate buffer, where IL concentration was assumed to be $0 \mathrm{wt} \%$ in this study. A cellulase solution was added to the biomass suspension at $40 \mathrm{FPU} / \mathrm{g}$-dry bagasse. The enzymatic hydrolysis reaction was conducted in $100-\mathrm{mL}$ vials at $50^{\circ} \mathrm{C}$ using a reciprocal shaker at $130 \mathrm{rpm}$.

During the enzymatic reaction, samples were collected after $0,3,6,9,12,24$, and $48 \mathrm{~h}$, and then heated at $90^{\circ} \mathrm{C}$ for 5 min to inactivate the enzyme. After centrifugation of the heated sample at 21,500 $\times \mathrm{g}$ for $1 \mathrm{~min}$, the glucose and xylose concentrations in the hydrolysate were determined by high-performance liquid chromatography (HPLC), as described below. Cellulose saccharification was evaluated as the percentage of cellulose hydrolyzed into glucose from cellulose in the original bagasse. In the same manner, hemicellulose saccharification was evaluated as the percentage of hemicellulose hydrolyzed into xylose from hemicellulose in the original bagasse.

\subsection{Electrodialysis-assisted separation of IL and monosaccharide}

An electrodialysis system (DW-Lab, AGC Engineering. Co., Ltd, Chiba, Japan) was used to remove salt, in this case IL, from the in situ enzymatic hydrolysate, which contains soluble sugars and IL in a buffer solution. The electrodialysis system comprises a membrane stack, circulation pumps for each of three compartments (desalination tank, concentration tank, and electrolyte tank: working volumes are all $250 \mathrm{~mL}$ ), and a DC power supply (PMC18-3A, Kikusui electronics Corp., Yokohama, Japan) (Fig. S1). The 
membrane stack has 5 pairs of anion-exchange and cation-exchange membranes (SELEMION $^{\circledR}$, AGC Engineering. Co., Ltd) with an effective area of $50 \times 60 \mathrm{~mm}$. For the desalination tank, the hydrolysate of the in situ enzymatic saccharification (containing the soluble sugars and the IL) was added to the solution to be desalinated, where the insoluble saccharification residue (mainly lignin) was in advance removed from the hydrolysate. For the concentration tank, $250 \mathrm{~mL}$ of distilled water was added as the initial solution. For the electrolyte tank, $250 \mathrm{~mL}$ of $\mathrm{Na}_{2} \mathrm{SO}_{4}$ aqueous solution (4 wt\%) was added as an electrode rinse solution. These three solutions were circulated at a flow rate of $4 \mathrm{~L} / \mathrm{min}$, respectively. The applied voltage was set to be $8 \mathrm{~V}$ throughout the operation.

During the electrodialysis, samples were collected from the desalination tank. The glucose and xylose concentrations in the desalination tank were determined by HPLC, as described below. IL (ChOAc) concentrations in the desalination tank were also determined by HPLC.

\subsection{Compositional analysis of biomass}

The cellulose, hemicellulose, and lignin contents of the original bagasse were determined according to the NREL method (Sluiter, et al., 2012) with minor modifications. In brief, $0.1 \mathrm{~g}$ of the sample was mixed with $2 \mathrm{~mL}$ of a $72 \%(v / v) \mathrm{H}_{2} \mathrm{SO}_{4}$ aqueous solution for $2 \mathrm{~h}$ at RT. The mixture was transferred to a 200-mL Erlenmeyer flask, diluted with $75 \mathrm{~mL}$ of water, and autoclaved at $121^{\circ} \mathrm{C}$ for $15 \mathrm{~min}$. The acid-diluted hydrolysate was filtered, after which the amount of acid-insoluble lignin was gravimetrically determined by measuring the residue on the filter after drying at $100^{\circ} \mathrm{C}$ for $12 \mathrm{~h}$. The amount of acid-soluble lignin was determined from the ultraviolet 
(UV) absorbance of the filtrate at $205 \mathrm{~nm}$ and an absorption coefficient of $110 \mathrm{~L} \mathrm{~g}^{-1}$ $\mathrm{cm}^{-1}$. The sum of the acid-insoluble and acid-soluble lignin was regarded as the total lignin amount. The amount of sugars in the hydrolysate was determined by HPLC equipped with a refractive index (RI) detector (Shimadzu Co., Kyoto, Japan) using a CARBOSep CHO-682 column (Tokyo Chemical Industry Co., Ltd, Tokyo, Japan). The column was run at $85^{\circ} \mathrm{C}$ with a water mobile phase and a flow rate of $0.4 \mathrm{~mL} / \mathrm{min}$. The amount of cellulose and hemicellulose was calculated from the glucose and xylose content multiplied by anhydro correction factors of 162/180 and 132/150, respectively.

\subsection{Powder X-ray diffractometry (PXRD)}

For PXRD, the biomass (bamboo powder, microcrystalline cellulose, or alkali lignin) was scanned using a horizontal X-ray diffractometer equipped with $\mathrm{Cu}-\mathrm{K} \alpha$ radiation (Ultima IV, Rigaku Corporation. Tokyo, Japan) over the $2 \theta$ range $5-40^{\circ}$ with a scan step of $0.05^{\circ}$. The acceleration voltage and current were $40 \mathrm{kV}$ and $30 \mathrm{~mA}$, respectively. The background intensity without the sample was subtracted from the intensity obtained for respective samples. The crystallinity index (CrI) was calculated from PXRD data according to the peak-height method using the following equation:

$$
\underline{\mathrm{CrI}}=\underline{\frac{I_{002}-I_{\mathrm{am}}}{I_{002}} \times 100}
$$

where $\underline{I}_{002}$ and $\underline{I}_{\mathrm{am}}$ are the maximum intensity of the (002) lattice diffraction and the minimum intensity between the (101) and (002) lattice planes, respectively.

\section{Results and discussion}

3.1. Pretreatment capability of ChOAc or EmimOAc for enzymatic hydrolysis of bagasse 
To examine the pretreatment capability of ChOAc or EmimOAc for subsequent enzymatic hydrolysis of lignocellulosic biomass, bagasse powder was pretreated in each IL and then hydrolyzed by Ctec 2 cellulase for $48 \mathrm{~h}$, where the IL was washed out from the pretreated biomass before saccharification to eliminate any inhibitory effect of IL on the cellulolytic enzyme. Figure 1 shows the time profile for the enzymatic reaction of pretreated bagasse up to $48 \mathrm{~h}$. Without IL pretreatment, cellulose and hemicellulose saccharification percentages were $24 \%$ and $9 \%$ of the theoretical maximum at $48 \mathrm{~h}$. In contrast, with ChOAc pretreatment, cellulose and hemicellulose saccharification percentages increased with an elapsed enzymatic reaction time, reaching $85 \%$ and $100 \%$ of the theoretical maximum at $48 \mathrm{~h}$. These time profiles are approximately in line with the cases using EmimOAc, although the cellulose saccharification percentage was somewhat less than the case of EmimOAc. These results indicated that ChOAc had a sufficient pretreatment capability for lignocellulosic materials, which was at almost the same level as EmimOAc under the examined pretreatment condition.

To examine the cellulose crystallinity in the bagasse powder pretreated in ChOAc or EmimOAc with ultrasound irradiation, XRD analysis was conducted and the CrI was determined on the basis of the XRD spectra. The CrI values were $61.9 \%, 49.1 \%$, and 41.7\% for the original biomass, biomass pretreated in ChOAc, and biomass pretreated in EmimOAc, respectively (Table S1). These results was roughly consistent with the cellulose saccharification results shown in Fig 1A, suggesting that ChOAc had a substantial pretreatment capability for lignocellulosic biomass

EmimOAc has been frequently thought of as the most effective IL for cellulose dissolution and pretreatment of enzymatic hydrolysis (Brandt, et al., 2013). Pretreatment with ChOAc enhanced the cellulose saccharification to the same degree as pretreatment 
with EmimOAc (Fig 1), suggesting that the acetate anion has an important role in the reduction of cellulose crystallinity. In general, anions act as hydrogen bond acceptors that interact with the hydroxyl group of cellulose, thereby weakening the crystalline structure of cellulose (Remsing, et al., 2006), whereas cations interact with lignin through hydrogen bonding and $\pi-\pi$ interactions (Janesko, 2011). In contrast, the inhibitory effects of ChOAc on cellulase were significantly lower than that of EmimOAc (Fig. 2), because the cholinium cation was reported to be more biocompatible than the imidazolium cation (Ninomiya, 2013; Petkovic, et al., 2010; Hou, et al., 2013). Therefore, it is suggested that ChOAc is a promising alternative to EmimOAc for the IL pretreatment and for in situ saccharification of lignocellulosic biomass.

\subsection{Inhibitory effect of ChOAc or EmimOAc on cellulolytic enzyme}

To examine the inhibitory effect of ChOAc or EmimOAc on cellulase enzyme, enzymatic saccharification of the PASC as amorphous cellulose was conducted for $24 \mathrm{~h}$ using Ctec2 cellulase in the presence of the prescribed concentration of each IL. Figure 2 shows dose-response relationships between PASC saccharification activity and IL concentration. ChOAc shows a significantly smaller inhibitory effect on cellulase compared with EmimOAc. The half maximal (50\%) inhibitory concentration (IC50) for Ctec2 cellulase was approximately $32 \mathrm{wt} \%$ and $16 \mathrm{wt} \%$ for ChOAc and EmimOAc, respectively. Thus, it was found that ChOAc shows less of an inhibitory effect on cellulase compared with EmimOAc under the examined conditions.

In general, cellulase enzymes in the IL aqueous solution lose their activity due to a higher salt concentration and ionic strength. Thus, a more biocompatible IL has been 
screened so as to perform the in situ enzymatic saccharification effectively. As the biocompatible candidate in imdazolium IL, MmimDMP was often studied with regard to its pretreatment capability of biomass (Yang, et al., 2010; Li, et al., 2010), its inhibitory effect on cellulase (Li, et al., 2010; Engel, et al., 2010; Wahlström, et al., 2010), and its application to in situ saccharification processes (Yang, et al., 2010; Li, 2010; Feng, et al., 2011; Abels, et al., 2013). On the other hand, the present study for the first time compared the cholinium IL (ChOAc) and the imidazolium IL (EmimOAc) in terms of the pretreatment capability of biomass (Fig. 1), the inhibitory effects on cellulase (Fig. 2) and the performance of in situ enzymatic saccharification (Fig. 3). Some researchers (Wahlström, et al., 2014) demonstrated that MmimDMP has a smaller inhibitory effect on cellulase than EmimOAc using the endoglucanases (such as Cel7B or Cel5A) and microcrystalline cellulose, which were different experimental conditions from the present study. There have been no reports on a direct comparison between ChOAc and MmimDMP in terms of inhibitory effects on cellulase enzymes and in situ saccharification performance under the same conditions (type of cellulase and substrate, cellulase loading to biomass, cellulase reaction time, i.e., exposure time to IL, and so on). We are now investigating this point to explore the appropriate IL for in situ saccharification. Moreover, it is necessary to employ an IL-tolerant cellulase such as thermophilic cellulase (Datta, et al., 2010; Shi, et al., 2013) as well as biocompatible IL for further reduction of water use for diluting IL prior to in situ saccharification.

\subsection{IL pretreatment and in situ saccharification using ChOAc or EmimOAc}

To demonstrate the IL pretreatment and in situ enzymatic saccharification of lignocellulosic biomass using ChOAc or EmimOAc, bagasse powder was pretreated in 
each IL and then in situ hydrolyzed by Ctec2 cellulase for $48 \mathrm{~h}$ after diluting to the prescribed IL concentration without washing out the IL from the pretreated biomass. Figure 3 shows the cellulose and hemicellulose saccharification percentage obtained at $48 \mathrm{~h}$ of the in situ enzymatic saccharification with different IL concentrations. In the cases for ChOAc, the cellulose and hemicellulose saccharification percentages were larger under the examined IL concentrations, ranging from 10 to 40\%. When the in situ saccharification was conducted in the presence of 10\% ChOAc, the cellulose and hemicellulose saccharification percentages were $80 \%$ and $72 \%$, respectively. In the case of $20 \%$ ChOAc, the cellulose and hemicellulose saccharification percentages reached $72 \%$ and 53\%, respectively. In contrast, in the case of 20\% EmimOAc, the cellulose and hemicellulose saccharification percentages were just $28 \%$ and $2 \%$, respectively. Thus, it was found that ChOAc was more suitable for the in situ enzymatic saccharification process than EmimOAc.

The biomass-to-sugar conversion process employed in this study has been referred as “IL pretreatment and in situ saccharification” or “one-pot IL pretreatment and saccharification.” The present study is the first to demonstrate ChOAc showing both biomass pretreatment capability and biocompatibility for cellulase, and then to apply ChOAc to the in situ saccharification of lignocellulosic material. This in situ saccharification eliminates the conventional step of washing out IL from the pretreated biomass, which can reduce the amount of wastewater and can prevet a "wash out" of soluble oligosaccharides generated during the biomass pretreatment with IL (typically seen in the case of EmimOAc (Table S1)). However, according to the techno-economic analysis for the overall process of in situ saccharification (Konda, et al., 2014), it is needed to satisfy the high biomass loading (33-50 wt\%) during the pretreatment step as 
well as the low IL cost (\$2.5-0.7/kg) and the high IL recovery (97-99.6\%) for ensuring economic sustainability. Our previous study (Ninomiya, et al., 2013a) revealed that ChOAc pretreatment at a biomass loading of $25 \mathrm{wt} \%$ (biomass: IL = 1: 3) did not deteriorate pretreatment efficiency (i.e., cellulose saccharification), but the cellulose saccharification percentage decreased to $50-60 \%$ when biomass loading was increased to $50 \mathrm{wt} \%$ (biomass: $\mathrm{IL}=1: 1$ ). Thus, this is required to enhance pretreatment efficiency even when the biomass loading is $50 \mathrm{wt} \%$ by a combined use of IL and microwave irradiation. This process will make the in situ saccharification process more economical and environmentally friendly due to less IL use and less wastewater discharge.

\subsection{Removal of IL from hydrolysate of in situ saccharification by electrodialysis}

To obtain the monosaccharide solution free from IL after the in situ enzymatic saccharification, electrodialysis was performed to remove IL from the hydrolysate of in situ saccharification, containing soluble sugars and ChOAc. Figure 4 shows the time courses of glucose, xylose, and ChOAc concentrations in the desalination tank during electrodialysis. The hydrolysate was obtained from the in situ enzymatic saccharification at $10 \mathrm{wt} \%$ of ChOAc concentration. ChOAc concentration decreased with an elapsed time of electrodialysis, reaching almost zero at 72 min. Glucose and xylose concentrations did not decrease, and rather slightly increased due to the removal of ChOAc from the hydrolysate. In contrast, in the concentration tank of the electrodialyzer, glucose and xylose were not detected even after 120 min of electrodialysis. The recovery percentage of ChOAc was $99 \%$ at the 120 min point. Thus, it was found that a monosaccharide solution free from IL and IL aqueous solution without soluble sugars could be obtained through electrodialysis of the hydrolysate of 
the in situ enzymatic saccharification.

The in situ saccharification is free from the washing out of IL from the pretreated biomass, and instead requires the separation of IL from the hydrolysate of the pretreated biomass. To date, to separate IL from the hydrolysate of in situ enzymatic (or chemical) saccharification, various principles have been employed such as ion-exclusion chromatography (Li, et al., 2010; Feng, et al., 2011; Sun, et al., 2013), electrodialysis with an ion-exchange membrane (Abels, et al., 2013), liquid-liquid extraction (Li, et al., 2012; Shi, et al., 2013), and an aqueous biphasic system using kosmotropic salts (Sun, et al., 2013). In the present study, the electrodialysis method was selected (Fig. 4), because it does not require any extra reagents such as acids and bases used in the phase separation described above (Li, et al., 2012; Sun, et al., 2013). Moreover, the electrodialysis method itself has already been employed in the salt production industry, although chromatography was unsuitable for scale-up to an industrial level. The present study did not concucted a separation and recovery of cellulase enzyme from the sugar solution. On this point, some researchers have demonstrated recovery of enzyme and IL by means of ultrafiltration and subsequent electrodialysis, respectively, after the the in situ saccharification of cellulosic material (Abels, et al., 2013). Now, we are going to develop the ChOAc-assisted in situ saccharification of lignocellulosic biomass accompanied with enzyme and IL recovery.

\section{Conclusions}

ChOAc, which showed a biomass pretreatment capability and a smaller inhibitory effect on cellulase than EmimOAc, was used for IL/ultrasound-assisted pretreatment and in situ enzymatic saccharification. The cellulose and hemicellulose saccharification 
percentage was $80 \%$ and $72 \%$, respectively, when the in situ saccharification was performed for $48 \mathrm{~h}$ in the presence of $10 \%$ ChOAc. The cellulose and hemicellulose saccharification percentage reached $72 \%$ and $53 \%$, respectively, even in the case of $20 \%$ ChOAc. A glucose/xylose solution free from IL and ChOAc aqueous solution without these sugars could be recovered separately by electrodialysis of the hydrolysate of the in situ enzymatic saccharification.

\section{Appendix A. Supplementary data}

Supplementary data associated with this article can be found, in the online version, at \#\#\#\#\#\#\#\#\#\#\#\#\#\#

\section{Acknowledgments}

This study was supported in part by an Advanced Low Carbon Technology Research and Development Program (ALCA) (Grant number 2100040) from Japan Science and Technology Agency. This study was supported in part by a Center of Innovation Science and Technology based Radical Innovation and Entrepreneurship Program (COIstream) from Japan Science and Technology Agency. This study was supported in part by a Cross-ministerial Strategic Innovation Promotion Program (SIP) from Japan Science and Technology Agency.

\section{References}

1. Abels, C., Thimm, K., Wulfhorst, H., Spiess, A.C., Wessling, M., 2013.

Membrane-based recovery of glucose from enzymatic hydrolysis of ionic liquid pretreated cellulose, Bioresour. Technol. 149, 58-64. 
2. Adney, B., Baker, J., 2008. Measurement of cellulase activities, Technical Report, NREL/TP-510-42628, National Renewable Energy Laboratory, Golden, CO.

3. Binder, J.B., Raines, R.T., 2010. Fermentable sugars by chemical hydrolysis of biomass. Proc. Natl. Acad. Sci. U. S. A. 107, 4516-4521.

4. Brandt, A., Gräsvik, J., Hallett, J.P., Welton, T., 2013. Deconstruction of lignocellulosic biomass with ionic liquids, Green Chem. 15, 550-583.

5. Dadi, A.P., Varanasi, S., Schall, C.A., 2006. Enhancement of cellulose saccharification kinetics using an ionic liquid pretreatment step, Biotechnol. Bioeng. 95, 904-910.

6. Datta, S., Holmes, B., Park, J.I., Chen, Z., Dibble, D.C., Hadi, M., Blanch, H.W., Simmons, B.A., Sapra, R., 2010. Ionic liquid tolerant hyperthermophilic cellulases for biomass pretreatment and hydrolysis. Green Chem. 12, 338-345.

7. Engel, P., Mladenov, R., Wulfhorst, H., Jäger, G., Spiess, A.C., 2010. Point by point analysis: how ionic liquid affects the enzymatic hydrolysis of native and modified cellulose. Green Chem. 12, 1959-1966.

8. Feng, D., Li, L. Yang, F., Tan, W., Zhao, G., Zou, H., Xian, M., Zhang, Y., 2011. Separation of ionic liquid [Mmim][DMP] and glucose from enzymatic hydrolysis mixture of cellulose using alumina column chromatography. Appl. Microbiol. Biotechnol. 91, 399-405.

9. Fukaya, Y., Iizuka, Y., Sekikawa, K., Ohno, H., 2007. Bio ionic liquids: room temperature ionic liquids composed wholly of biomaterials. Green Chem. 9, 11551157.

10. Hou, X.D., Liu, Q.P., Smith, T.J., Li, N., Zong, M.H., 2013. Evaluation of toxicity and biodegradability of cholinium amino acids ionic liquids. PLoS One. 8, e59145. 
11. Hu, S., Jiang, T., Zhang, Z., Zhu, A., Han, B., Song, J., Xie, Y., Li, W., 2007. Functional ionic liquid from biorenewable materials: synthesis and application as a catalyst in direct aldol reactions. Tetrahedron Lett. 48, 5613-5617.

12. Janesko, B.G., 2011. Modeling interactions between lignocellulose and ionic liquids using DFT-D. Phys. Chem. Chem. Phys. 13, 11393-11401.

13. Kamiya, N., Matsushita, Y., Hanaki, M., Nakashima, K., Narita, M., Goto, M., Takahashi, H., 2008. Enzymatic in situ saccharification of cellulose in aqueous-ionic liquid media. Biotechnol. Lett. 30, 1037-1040.

14. Konda, N.M., Shi, J., Singh, S., Blanch, H.W., Simmons, B.A., Klein-Marcuschamer, D., 2014. Understanding cost drivers and economic potential of two variants of ionic liquid pretreatment for cellulosic biofuel production. Biotechnol. Biofuels. 7, 86.

15. Li, C., Knierim, B., Manisseri, C., Arora, R., Scheller, H.V., Auer, M., Vogel, K.P., Simmons, B.A., Singh, S., 2010. Comparison of dilute acid and ionic liquid pretreatment of switchgrass: Biomass recalcitrance, delignification and enzymatic saccharification. Bioresour. Technol. 101, 4900-4906.

16. Li, Q., Jiang, X., He, Y., Li, L., Xian, M., Yang, J., 2010. Evaluation of the biocompatibile ionic liquid 1-methyl-3-methylimidazolium dimethylphosphite pretreatment of corn cob for improved saccharification. Appl. Microbiol. Biotechnol. 87, 117-126.

17. Li, C., Cheng, G., Balan, V., Kent, M.S., Ong, M., Chundawat, S.P.S., Sousa, L.C., Melnichenko, Y.B., Dale, B.E., Simmons, B.A., Singh, S., 2011. Influence of physico-chemical changes on enzymatic digestibility of ionic liquid and AFEX pretreated corn stover. Bioresour. Technol. 102, 6928-6936. 
18. Liu, Q.P., Hou, X.D., Li, N., Zong, M.H., 2012. Ionic liquids from renewable biomaterials: synthesis, characterization and application in the pretreatment of biomass. Green Chem. 14, 304-307.

19. Li, L., Xie, J., Yu, S., Su, Z., Liu, S., Liu, F., Xie, C., Zhang, B., 2012. Novel compatible system of [ $\left.\mathrm{C}_{2} \mathrm{OHmim}\right][\mathrm{OAc}]$-cellulases for the in situ hydrolysis of lignocellulosic biomass. RSC Adv. 2, 11712-11718.

20. Ninomiya, K., Soda, H., Ogino, C., Takahashi, K., Shimizu, N., 2013a. Effect of ionic liquid weight ratio on pretreatment of bamboo powder prior to enzymatic saccharification. Bioresour. Technol. 128, 188-192.

21. Ninomiya, K., Yamauchi, T., Kobayashi, M., Chiaki, O., Shimizu, N., Takahashi, K., 2013b. Cholinium carboxylate ionic liquids for pretreatment of lignocellulosic materials to enhance subsequent enzymatic saccharification. Biochem. Eng. J. 71, $25-29$.

22. Ninomiya, K., Ohta, A., Omote, S., Ogino, C., Takahashi, K., Shimizu. N., 2013c. Combined use of completely bio-derived cholinium ionic liquids and ultrasound irradiation for the pretreatment of lignocellulosic material to enhance enzymatic saccharification, Chem. Eng. J., 215-216, 811-818.

23. Ouellet, M., Datta, S., Dibble, D.C., Tamrakar, P.R., Benke, P.I., Li, C., Singh, S., Sale, K.L., Adams, P.D., Keasling, J.D., Simmons, B.A., Holmes, B.M., Mukhopadhyay, A., 2011. Impact of ionic liquid pretreated plant biomass on Saccharomyces cerevisiae growth and biofuel production. Green Chem. 13, 27432749.

24. Petkovic, M., Ferguson, J.L., Gunaratne, H.N., Ferreira, R., Leitao, M.C., Seddon, K.R., Rebelo, L.P.N., Silva Pereira, C., 2010. Novel biocompatible cholinium-based 
ionic liquids - Toxicity and biodegradability. Green Chem. 12, 643-649.

25. Remsing, R.C., Swatloski, R.P., Rogers, R.D., Moyna, G., 2006. Mechanism of cellulose dissolution in the ionic liquid 1-n-butyl-3-methylimidazolium chloride: a ${ }^{13} \mathrm{C}$ and ${ }^{35 / 37} \mathrm{Cl}$ NMR relaxation study on model systems. Chem. Commun. $1271-$ 1273.

26. Shi, J., Gladden, J.M., Sathitsuksanoh, N., Kambam, P., Sandoval, L., Mitra, D., Zhang, S., George, A., Singer, S.W., Simmons, B.A., Singh, S., 2013. One-pot ionic liquid pretreatment and saccharification of switchgrass. Green Chem. 15, 25792589.

27. Sun, N., Liu, H., Sathitsuksanoh, N., Stavila, V., Sawant, M., Bonito, A., Tran, K., George, A., Sale, K.L., Singh, S., Simmons, B.A., Holmes, B.M., 2013. Production and extraction of sugars from switchgrass hydrolyzed in ionic liquids. Biotechnol. Biofuels. 6, 39.

28. Sluiter, A., Hames, B., Ruiz, R., Scarlata, C., Sluiter, J., Templeton, D., Crocker, D., 2012. Determination of structural carbohydrates and lignin in biomass determination of structural carbohydrates and lignin in biomass, Technical Report, NREL/TP-510-42618, National Renewable Energy Laboratory, Golden, CO.

29. Swatloski, R.P., Spear, S.K., Holbrey, J.D., Rogers, R.D., 2002. Dissolution of cellose with ionic liquids. J. Am. Chem. Soc. 124, 4974-4975.

30. Wahlström, R., Rahikainen, J., Kruus, K., Suurnäkki, A., 2014. Cellulose hydrolysis and binding with Trichoderma reesei Cel5A and Cel7A and their core domains in ionic liquid solutions. Biotechnol. Bioeng. 111, 726-733.

31. Wang, Y., Radosevich, M., Hayes, D., Labbé, N., 2011. Compatible ionic liquid-cellulases system for hydrolysis of lignocellulosic biomass, Biotechnol. 
Bioeng. 108, 1042-1048.

32. Wood, T.M., 1988. Preparation of crystalline, amorphous, and dyed cellulase substrates, Methods Enzymol. 160, 19-25.

33. Xu, J., He, B., Wu, B., Wang, B., Wang, C., Hu. L., 2014. An ionic liquid tolerant cellulase derived from chemically polluted microhabitats and its application in in situ saccharification of rice straw. Bioresour. Technol. 157, 166-173.

34. Yang, F., Li, L., Li, Q., Tan, W., Liu, W., Xian, M., 2010. Enhancement of enzymatic in situ saccharification of cellulose in aqueous-ionic liquid media by ultrasonic intensification. Carbohydr. Polym. 81, 311-316.

35. Yu, Y., Lu, X., Zhou, Q., Dong, K., Yao, H., Zhang, S., 2008. Biodegradable naphthenic acid ionic liquids: synthesis, characterization, and quantitative structure-biodegradation relationship. Chem. Eur. J. 14, 11174-11782. 


\section{Figure captions}

Fig. 1 Time courses of saccharification percentage for cellulose (A) and hemicellulose (B) during enzymatic reaction of bagasse powder pretreated with IL. Prior to the enzymatic reaction, IL was washed out from the pretreated bagasse powder. Closed circles: ChOAc, open circles: EmimOAc, gray circles: control without pretreatment. The error bars indicate the standard deviation from three independent experiments.

Fig. 2 Dose-response relationships between relative cellulase activity and IL concentration. Cellulase activity was evaluated by the amount of glucose generated from PASC for $24 \mathrm{~h}$ of enzymatic reaction in the presence of IL at the subscribed concentration, and expressed as a percentage of the value without IL addition. Closed circles: ChOAc, open circles: EmimOAc. The error bars indicate the standard deviation from three independent experiments.

Fig. 3 Saccharification percentages for cellulose (A) and hemicellulose (B) obtained at $48 \mathrm{~h}$ of the in situ saccharification of bagasse powder at the subscribed IL concentration in the saccharification step. Closed bars: ChOAc, open bars: EmimOAc, gray bars: control without pretreatment. The error bars indicate the standard deviation from three independent experiments.

Fig. 4 Time course of ChOAc, glucose, and xylose concentrations in the desalination tank during electrodialysis of hydrolysate obtained from the in situ 
saccharification of bagasse powder at 10\% ChOAc concentration for saccharification step. Closed circles: ChOAc, closed triangles: glucose, closed diamonds: xylose. 

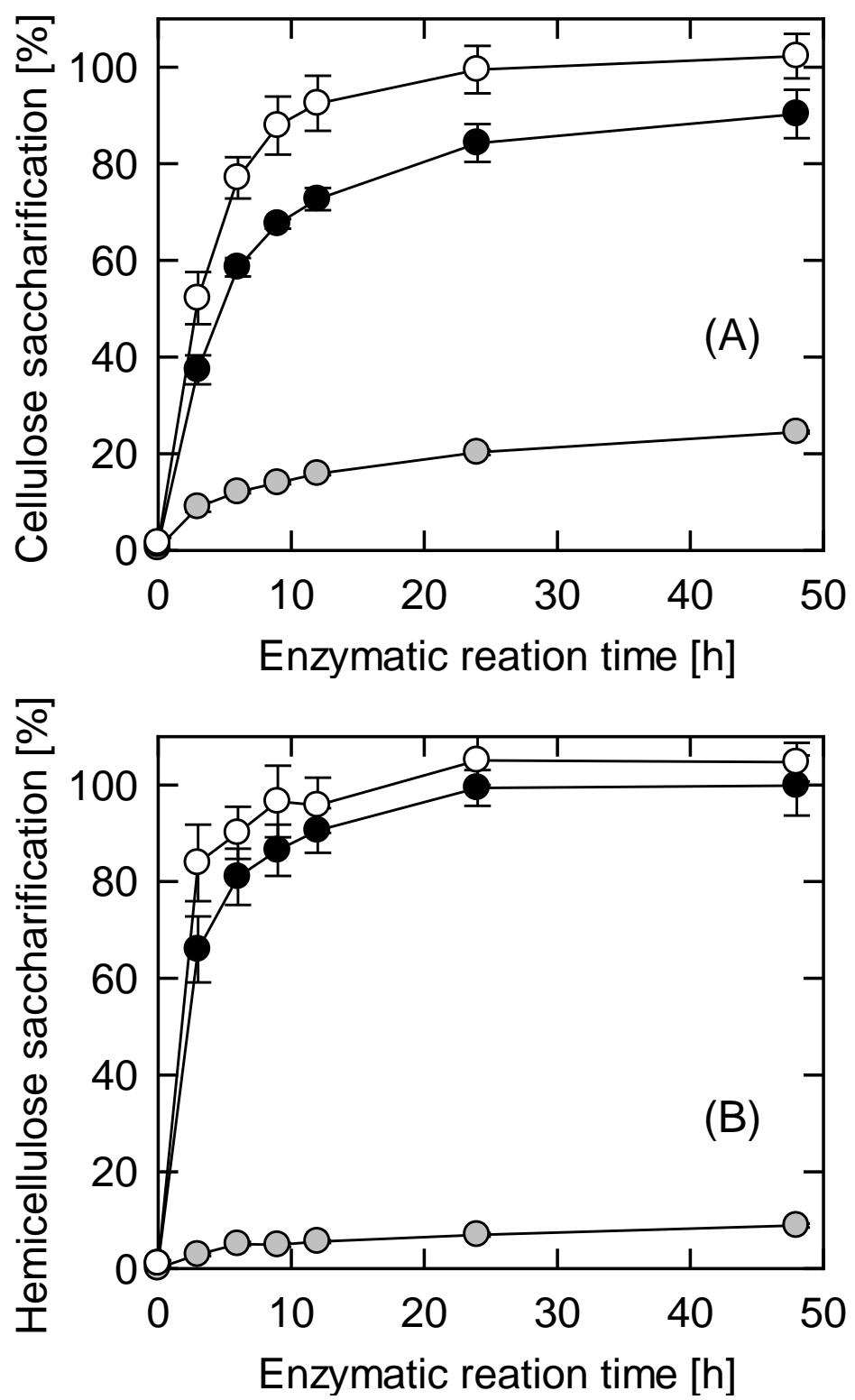

Fig. 1. 


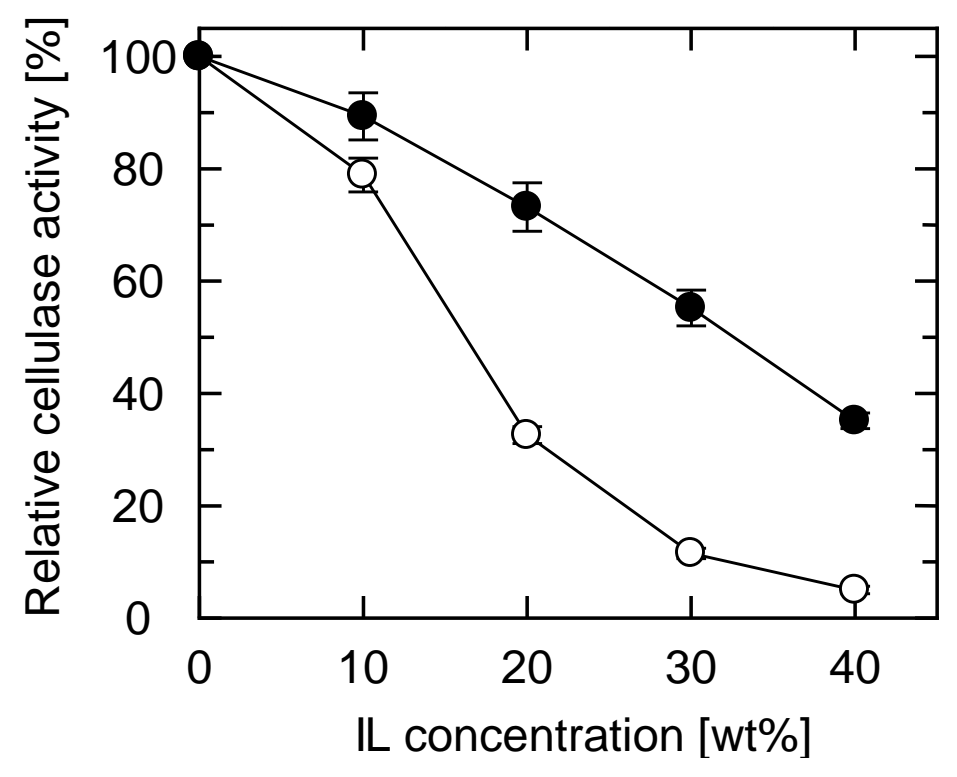

Fig. 2. 

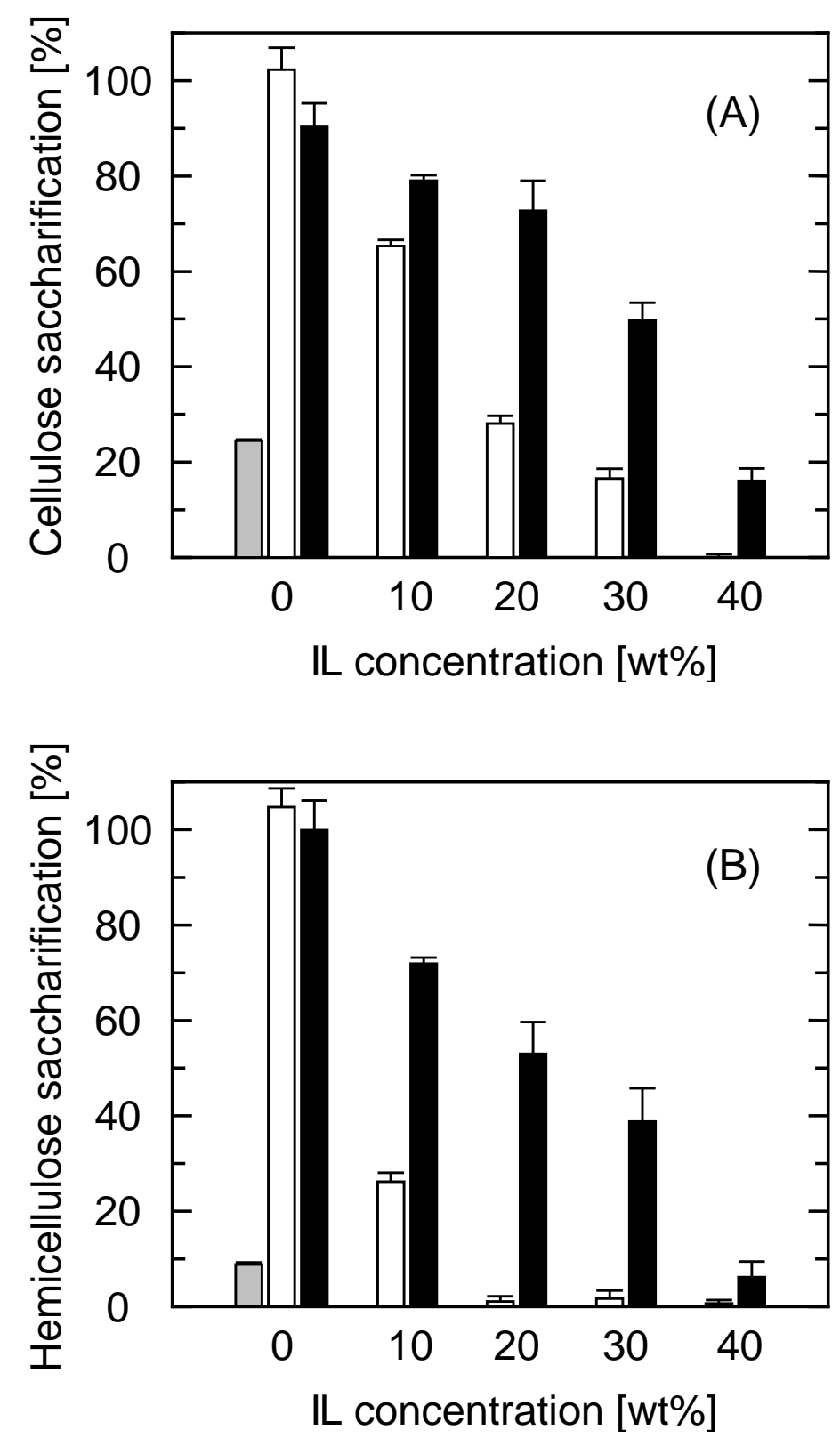

Fig. 3. 


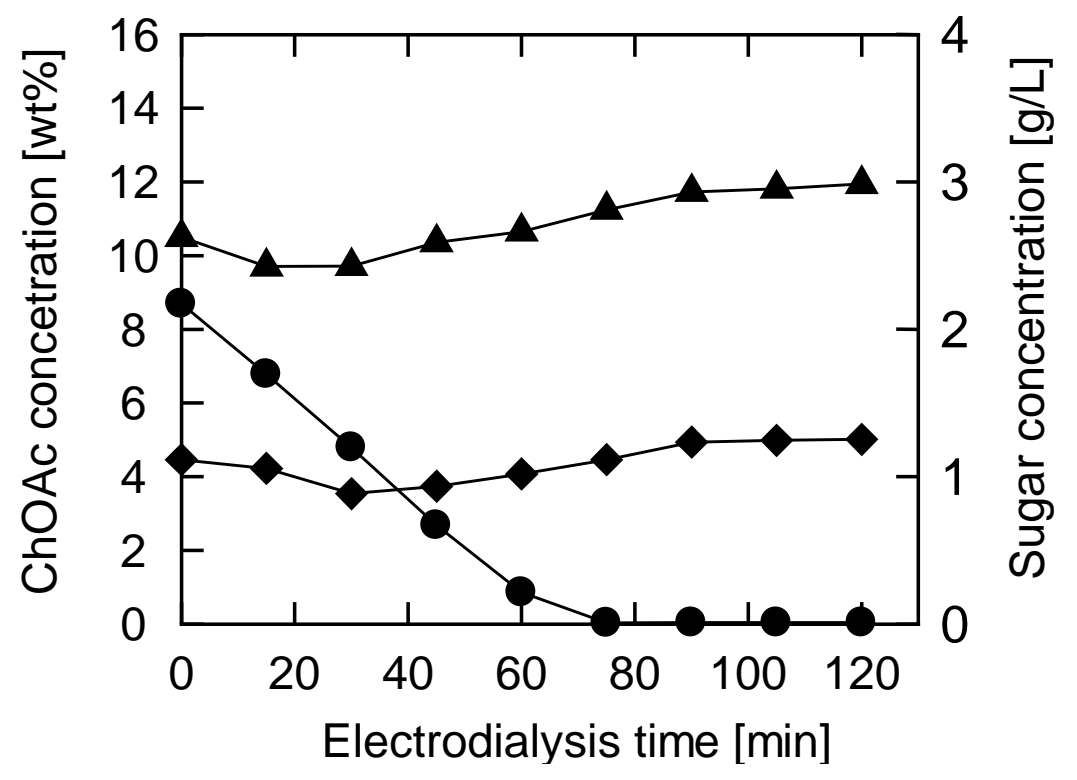

Fig. 4. 


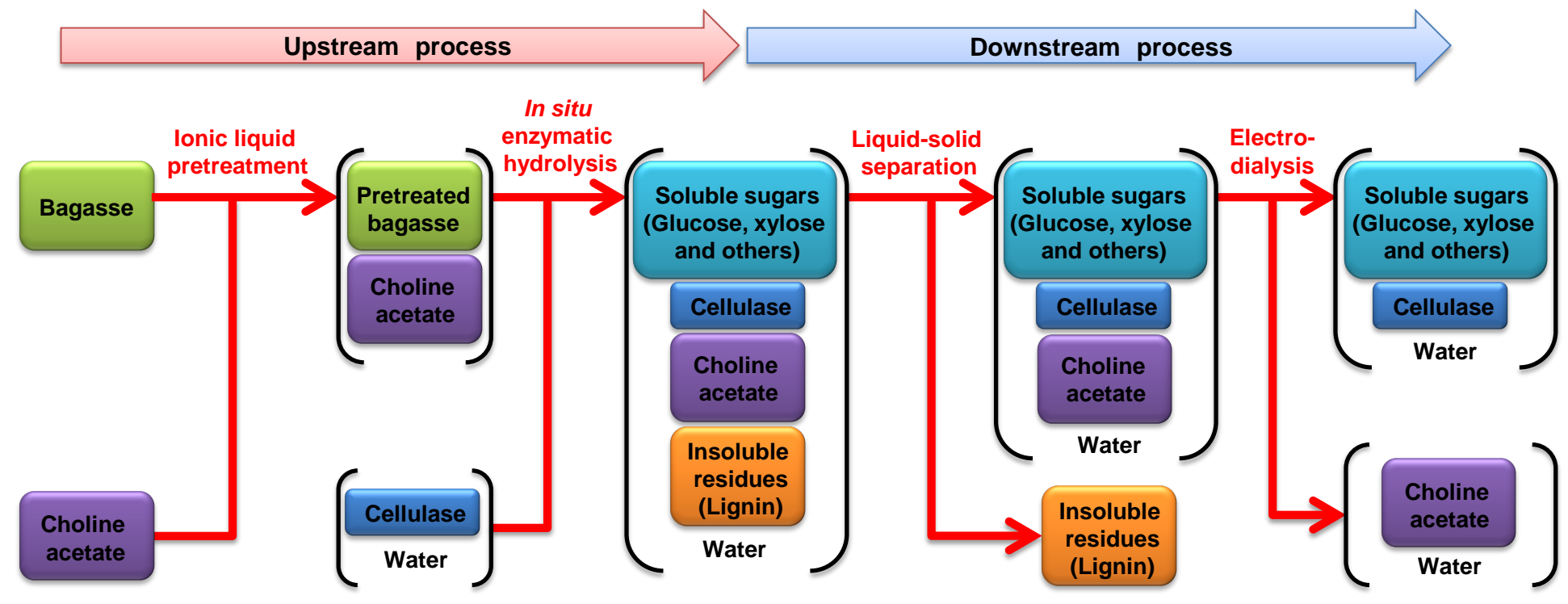

